\title{
Exact Multiplicity of Sign-Changing Solutions for a Class of Second-Order Dirichlet Boundary Value Problem with Weight Function
}

\author{
Yulian An \\ Department of Mathematics, Shanghai Institute of Technology, Shanghai 201418, China \\ Correspondence should be addressed to Yulian An; an_yulian@sit.edu.cn
}

Received 29 March 2013; Accepted 13 May 2013

Academic Editor: Maoan Han

Copyright (C) 2013 Yulian An. This is an open access article distributed under the Creative Commons Attribution License, which permits unrestricted use, distribution, and reproduction in any medium, provided the original work is properly cited.

Using bifurcation techniques and Sturm comparison theorem, we establish exact multiplicity results of sign-changing or constant sign solutions for the boundary value problems $u^{\prime \prime}+a(t) f(u)=0, t \in(0,1), u(0)=0$, and $u(1)=0$, where $f \in C(\mathbb{R}, \mathbb{R})$ satisfies $f(0)=0$ and the limits $f_{\infty}=\lim _{|s| \rightarrow \infty}(f(s) / s), f_{0}=\lim _{|s| \rightarrow 0}(f(s) / s) \in\{0, \infty\}$. Weight function $a(t) \in C^{1}[0,1]$ satisfies $a(t)>0$ on $[0,1]$.

\section{Introduction}

In this paper, we consider the existence and exact multiplicity of sign-changing solutions for the boundary value problem

$$
\begin{gathered}
u^{\prime \prime}+a(t) f(u)=0, \quad t \in(0,1), \\
u(0)=0, \quad u(1)=0,
\end{gathered}
$$

where $f \in C(\mathbb{R}, \mathbb{R})$ satisfies $f(0)=0$ and weight function $a(t) \in C^{1}[0,1]$ satisfies $a(t)>0$ on $[0,1]$. The existence and multiplicity of positive or sign-changing solutions of boundary value problems have been extensively studied in the literature, see [1-7] and references therein.

However, for most nonlinearities $f$, a full description for the positive or sign-changing solution sets of many boundary value problems remains open. For some different boundary value problems, $[8,9]$ studied the exact multiplicity of positive solutions by bifurcation techniques, and [10-12] discussed the bifurcation diagrams of positive solutions by analyzing corresponding time maps. Recently, multiplicity of positive solutions to boundary blow-up elliptic problems with sign-changing weights was considered by [13].
As for exact multiplicity of sign-changing solutions, only few papers considered this problem. In [14], Shi studied the problem

$$
\begin{gathered}
u^{\prime \prime}+\lambda f(u)=0, \quad t \in(0,1), \\
u(0)=0, \quad u(1)=0,
\end{gathered}
$$

under the conditions

(C1) $f \in C^{1}(\mathbb{R}, \mathbb{R})$ satisfies $f(0)=0, f^{\prime}(u)>0$ for $u \in \mathbb{R}$;

(C2) $f^{\prime}(u)>f(u) / u$, if $u \neq 0$;

(C3) the limit $f_{\infty}=\lim _{|s| \rightarrow \infty}(f(s) / s) \in(0, \infty)$.

By using the implicit function theorem and local bifurcation theorems, the author obtained a full description of the set of sign-changing solutions of (2) for all values of $\lambda$. The set consists of some curves which bifurcate from the trivial solution line $u=0$ and tend to infinity. Particularly, there is no any turning points on these curves. Thus, they obtained the exact number of sign-changing solutions of the problem (2) for every given $\lambda \in \mathbb{R}$. Bari and Rynne [15] considered the $2 m$ th $(m \geq 2)$ order boundary value problem

$$
\begin{gathered}
(-1)^{m} u^{(2 m)}(t)=\lambda g(u(t)) u(t), \quad t \in(0,1), \\
u^{(i)}(-1)=u^{(i)}(1)=0, \quad i=0, \ldots, m-1,
\end{gathered}
$$


where $\lambda$ is a positive parameter, and the function $g \in$ $C^{1}(\mathbb{R}, \mathbb{R})$ satisfies $g(0)>0, \pm g^{\prime}(\xi)>0$, for $\pm \xi>0$, and $\lim _{|\xi| \rightarrow \infty} g(\xi)=\infty$. They got results similar to those in [14].

The basic steps developed in $[14,15]$ to prove exact multiplicity of sign-changing solutions involve: showing any nontrivial solution of (2) (or (3)) to be nondegenerate and proving uniqueness of solution curve on which any nontrivial solution of (2) (or (3)) has certain zero point number.

For other works on the exact multiplicity of signchanging, see $[16-19,23]$. In $[16,18,23]$, the main tools are also bifurcation techniques. Time maps and exact multiplicity results of sign-changing solutions for one-dimensional prescribed mean curvature equations were considered by $[17,19]$. However, all equations that had been studied in these works do not contain weight function $a(t)$. Reference [20] discussed the existence and multiplicity of sign-changing solutions of some boundary value problems with weight functions. This work was extended to more general cases by [21] by shooting method and [22] by bifurcation method.

In this paper, we consider the exact multiplicity of signchanging solutions of (1). Compared with [14-19, 23], this paper considers the case that the nonlinearity contains a weight function $a(t) \neq 1, t \in[0,1]$. On the other hand, we discuss the case that $f_{0}, f_{\infty} \in\{0, \infty\}$ instead of $f_{0}, f_{\infty} \in$ $(0, \infty)$. The main difficulty is to show any nontrivial solution of (1) to be nondegenerate. We will introduce an auxiliary function $g(\cdot)$ to deal with it. The method is motivated by the proof of Lemma 2.6 in [24], where the authors study global positive curves for a class of two-point boundary value problems. Compared with [24], we discuss not only the exact multiplicity of positive solutions but also of sign-changing solutions of (1).

The organization of this paper is as follows. In Section 2, we introduce some notations needed in later sections. We prove our main results in Sections 3 and 4. In Section 3, we study the exact multiplicity of sign-changing solutions of (1) under the hypotheses $f_{0}=0$ and $f_{\infty}=\infty$. In Section 4 , we consider the exact multiplicity of constant sign solutions of (1) under the conditions $f_{0}=\infty$ and $f_{\infty}=0$.

\section{Some Notations}

For applying bifurcation theorem, we consider the auxiliary problem

$$
\begin{gathered}
u^{\prime \prime}+\mu a(t) u+a(t) f(u)=0, \quad t \in(0,1), \\
u(0)=0, \quad u(1)=0,
\end{gathered}
$$

where $\mu \in \mathbb{R}$ is a parameter.

Clearly, any solution of $(4)$ of the form $(0, u)$ corresponds to a solution $u$ of $(1)$. From $f(0)=0$, we know $u \equiv 0$ is a solution of (4) for any $\mu \in \mathbb{R}$, such solutions will be called trivial solutions.

We introduce some notations to describe the properties of solutions of (4). For a function $u \in C^{1}[0,1]$, if $u\left(x_{0}\right)=0$, then $x_{0}$ is a simple zero of $u$ if $u^{\prime}\left(x_{0}\right) \neq 0$. For any $k \in \mathbb{N}:=\{1,2, \ldots\}$ and any $v \in\{+,-\}$, we define sets $S_{k}^{v} \subset C^{2}[0,1]$ consisting of the functions $u \in C^{2}[0,1]$ with

(i) $u(0)=0, v u^{\prime}(0)>0$;

(ii) $u$ has only simple zeros in $[0,1]$ and has exactly $k-1$ zeros in $(0,1)$.

Obviously, the sets $S_{k}^{v}$ are open in $\left\{u \in C^{1}[0,1] \mid u(0)=\right.$ $u(1)=0\}$ and disjoint.

Suppose that $(\mu, u)$ is a solution of $(4)$. Then the corresponding linearized problem of (4) is

$$
\begin{gathered}
w^{\prime \prime}+\mu a(t) w+a(t) f^{\prime}(u) w=0, \quad t \in(0,1), \\
w(0)=0, \quad w(1)=0 .
\end{gathered}
$$

We call the solution $(\mu, u)$ is nondegenerate if (5) has no nontrivial solution; otherwise it is degenerate. Sometimes, we call a degenerate solution a turning point.

Consider the linear problem

$$
\begin{gathered}
\varphi^{\prime \prime}+\mu a(t) \varphi=0, \quad t \in(0,1), \\
\varphi(0)=0, \quad \varphi(1)=0 .
\end{gathered}
$$

Remark 1. Note that $a(t) \in C^{1}[0,1]$ and $a(t)>0$ on $[0,1]$. It is well known that the eigenvalues of (6) are given by

$$
0<\lambda_{1}<\lambda_{2}<\cdots<\lambda_{k}<\lambda_{k+1}<\cdots, \quad \lim _{k \rightarrow \infty} \lambda_{k}=\infty .
$$

For each $k \in \mathbb{N}, \lambda_{k}$ is algebraic simple and the corresponding normalized eigenfunction can be chosen $\varphi_{k} \in S_{k}^{+}$.

In this paper, we work in the following spaces:

$$
\begin{aligned}
& X=\left\{u \in C^{2}[0,1]: u(0)=u(1)=0\right\}, \\
& \|u\|_{X}=\max \left\{\|u\|_{\infty},\left\|u^{\prime}\right\|_{\infty},\left\|u^{\prime \prime}\right\|_{\infty}\right\}, \\
& E=\left\{u \in C^{1}[0,1]: u(0)=u(1)=0\right\}, \\
& \|u\|_{E}=\max \left\{\|u\|_{\infty},\left\|u^{\prime}\right\|_{\infty}\right\}, \\
& Y=C[0,1], \quad\|u\|_{Y}=\|u\|_{\infty},
\end{aligned}
$$

where $\|\cdot\|_{\infty}$ is the normal supnorm. Obviously, $\left(X,\|\cdot\|_{X}\right)$ and $\left(E,\|\cdot\|_{E}\right)$ are Banach spaces.

\section{The Main Results under $f_{0}=0, f_{\infty}=\infty$}

In the section, we assume

(H1) $f \in C^{1}(\mathbb{R}, \mathbb{R})$ satisfies $u f(u)>0$, if $u \neq 0$;

(H2) $f^{\prime}(u)>f(u) / u$, if $u \neq 0$;

(H3) the limits $f_{0}:=\lim _{|s| \rightarrow 0}(f(s) / s)=0$ and $f_{\infty}:=$ $\lim _{|s| \rightarrow \infty}(f(s) / s)=\infty$;

(H4) $a(t) \in C^{2}[0,1]$ satisfies $a(t)>0$ and $3\left(a^{\prime}(t)\right)^{2}<$ $2 a(t) a^{\prime \prime}(t)$, if $t \in[0,1]$. 
Note that (H1) ensures that the solution of the initial value problem

$$
\begin{gathered}
u^{\prime \prime}+\mu a(t) u+a(t) f(u)=0, \quad t \in(0,1), \\
u\left(t_{0}\right)=\alpha, \quad u^{\prime}\left(t_{0}\right)=\beta
\end{gathered}
$$

is not only existent but also unique on the whole interval $[0,1]$ for any $t_{0} \in[0,1]$ and $\alpha, \beta \in \mathbb{R}$. This fact will be used repeatedly in the following proof so, for brevity, it will be abbreviated to "IVPU".

Remark 2. The condition (H4) appeared firstly in [24]. There are many functions satisfying (H4). Let $a(t)=b(t)+c$, where $b(\cdot) \in C^{2}[0,1]$ with $b^{\prime \prime}(t)>0, b^{\prime}(t)<0$, for all $t \in[0,1], c$ is a large enough constant. It is easy to check that $a(\cdot)$ satisfies (H4).

Now, we give some important lemmas.

Lemma 3. Suppose $(\mu, u)$ is a nontrivial solution of (4). Then,

(i) $u \in S_{k}^{v}$ for some $k \in \mathbb{N}$ and $v \in\{+,-\}$;

(ii) the zeros of $u$ and the zeros of $u^{\prime}$ are separated as $\mu \geq 0$.

Proof. (i) Since $u$ is nontrivial, "IVPU" implies that all the zeros of $u$ are simple. Thus, (i) is true.

(ii) When $\mu \geq 0$, we have

$$
\begin{array}{ll}
u^{\prime \prime}=-\mu a(t) u-a(t) f(u) \leq 0, & \text { if } u \geq 0, \\
u^{\prime \prime}=-\mu a(t) u-a(t) f(u) \geq 0, & \text { if } u \leq 0 .
\end{array}
$$

This implies that the zeros of $u$ and the zeros of $u^{\prime}$ are separated. Since $u \in S_{k}^{v}$ for some $k \in \mathbb{N}$ and $v \in\{+,-\}$, we get $u$ has $k-1$ zero on $(0,1)$ and $u^{\prime}$ has exact $k$ zero on $(0,1)$.

Lemma 4. Suppose $\mu \geq 0$ and $(\mu, u)$ is a nontrivial solution of (4). Then $(\mu, u)$ is nondegenerate.

Proof. we need to show that (5) has only trivial solution. Consider the initial problem

$$
\begin{gathered}
w^{\prime \prime}+\mu a(t) w+a(t) f^{\prime}(u) w=0, \quad t \in(0,1), \\
w(0)=0, \quad w^{\prime}(0)=1 .
\end{gathered}
$$

Clearly, (11) has a unique solution $w_{0}$. For every solution $w$ of (5), there exists a unique constant $c \in \mathbb{R}$ such that $w=c w_{0}$. We claim that

$$
w_{0}(1) \neq 0 \text {. }
$$

If (12) holds, then we immediately have $w(1)=0$ if and only if $c=0$. That is, to say $w \equiv 0$. Then we will finish the proof. Now, we prove (12) is true.

Firstly, we show that there is at least one zero of $u^{\prime}$ between consecutive zeros of $w_{0}$.

Suppose $\alpha, \beta$ are consecutive zeros of $w_{0}$, that is, $w_{0}(\alpha)=$ $w_{0}(\beta)=0$. Without loss of generality, suppose $w_{0}(t)>0$, $t \in(\alpha, \beta)$. If $u^{\prime}$ has no zero on $(\alpha, \beta)$, we assume $u^{\prime}(t)>0$, $t \in(\alpha, \beta)$. (When $u^{\prime}(t)<0, t \in(\alpha, \beta)$, the proof is similar to the case $u^{\prime}(t)>0$.)

Note that $w_{0}(t)$ and $u^{\prime}(t)$ satisfy the following equations, respectively:

$$
\begin{gathered}
w_{0}^{\prime \prime}+\mu a(t) w_{0}+a(t) f^{\prime}(u) w_{0}=0, \\
\left(u^{\prime}\right)^{\prime \prime}+\mu a(t) u^{\prime}+a(t) f^{\prime}(u) u^{\prime} \\
=-\mu a^{\prime}(t) u-a^{\prime}(t) f(u) .
\end{gathered}
$$

Multiply (14) by $g(t) w_{0}$ and subtract from it (13) multiplied by $g(t) u^{\prime}$, with $g(t)>0$ to be specified. Then integrate over $(\alpha, \beta)$

$$
\begin{aligned}
& \int_{\alpha}^{\beta}\left(u^{\prime}\right)^{\prime \prime} g w_{0} d t-\int_{\alpha}^{\beta} w_{0}^{\prime \prime} g u^{\prime} d t \\
& +\int_{\alpha}^{\beta}(\mu u+f(u)) a^{\prime} g w_{0} d t=0 .
\end{aligned}
$$

We denote the left side of (15) by $I$ and a constant $-w_{0}^{\prime}(\beta) g(\beta) u^{\prime}(\beta)+w_{0}^{\prime}(\alpha) g(\alpha) u^{\prime}(\alpha)$ by $A$. Integrating by parts,

$$
\begin{aligned}
I= & \left.u^{\prime \prime} g w_{0}\right|_{\alpha} ^{\beta}-\int_{\alpha}^{\beta} u^{\prime \prime}\left[g^{\prime} w_{0}+g w_{0}^{\prime}\right] d t \\
& -\left.w_{0}^{\prime} g u^{\prime}\right|_{\alpha} ^{\beta}+\int_{\alpha}^{\beta} w_{0}^{\prime}\left[g^{\prime} u^{\prime}+g u^{\prime \prime}\right] d t \\
& +\int_{\alpha}^{\beta}(\mu u+f(u)) a^{\prime} g w_{0} d t \\
= & -w_{0}^{\prime}(\beta) g(\beta) u^{\prime}(\beta)+w_{0}^{\prime}(\alpha) g(\alpha) u^{\prime}(\alpha) \\
& +\int_{\alpha}^{\beta} u^{\prime}\left[g^{\prime \prime} w_{0}+g^{\prime} w_{0}^{\prime}\right] d t+\int_{\alpha}^{\beta} w_{0}^{\prime} g^{\prime} u^{\prime} d t \\
& +\int_{\alpha}^{\beta}(\mu u+f(u)) a^{\prime} g w_{0} d t \\
= & A+\int_{\alpha}^{\beta} u^{\prime} g^{\prime \prime} w_{0} d t+2 \int_{\alpha}^{\beta} u^{\prime} g^{\prime} w_{0}^{\prime} d t \\
& +\int_{\alpha}^{\beta}(\mu u+f(u)) a^{\prime} g w_{0} d t \\
= & A+\int_{\alpha}^{\beta} u^{\prime} g^{\prime \prime} w_{0} d t \\
& +2\left[\left.u^{\prime} g^{\prime} w_{0}\right|_{\alpha} ^{\beta}-\int_{\alpha}^{\beta} w_{0}\left[u^{\prime \prime} g^{\prime}+u^{\prime} g^{\prime \prime}\right] d t\right] \\
& +\int_{\alpha}^{\beta}(\mu u+f(u)) a^{\prime} g w_{0} d t \\
& +\int_{\alpha}^{\beta}(\mu u+f(u)) a^{\prime} g w_{0} d t \\
= & A-\int_{\alpha}^{\beta} u^{\prime} g^{\prime \prime} w_{0} d t-2 \int_{\alpha}^{\beta} w_{0} g^{\prime} u^{\prime \prime} d t \\
&
\end{aligned}
$$




$$
\begin{aligned}
= & A-\int_{\alpha}^{\beta} u^{\prime} g^{\prime \prime} w_{0} d t-2 \int_{\alpha}^{\beta} w_{0} g^{\prime}(-\mu a u-a f(u)) d t \\
& +\int_{\alpha}^{\beta}(\mu u+f(u)) a^{\prime} g w_{0} d t \\
= & A-\int_{\alpha}^{\beta} u^{\prime} g^{\prime \prime} w_{0} d t+2 \int_{\alpha}^{\beta}(\mu u+f(u)) a^{\prime} g w_{0} d t \\
& +\int_{\alpha}^{\beta}(\mu u+f(u)) a^{\prime} g w_{0} d t \\
= & A-\int_{\alpha}^{\beta} u^{\prime} g^{\prime \prime} w_{0} d t \\
& +\int_{\alpha}^{\beta}(\mu u+f(u))\left(2 g^{\prime} a+a^{\prime} g\right) w_{0} d t .
\end{aligned}
$$

Let

$$
2 g^{\prime} a+a^{\prime} g=0, \quad g^{\prime \prime}<0
$$

on $(0,1)$. By the above supposition, we have

$$
I=-w_{0}^{\prime}(\beta) g(\beta) u^{\prime}(\beta)+w_{0}^{\prime}(\alpha) g(\alpha) u^{\prime}(\alpha)-\int_{\alpha}^{\beta} u^{\prime} g^{\prime \prime} w_{0} d t
$$$$
>0 \text {. }
$$

However, the right side of (15) is zero. A contradiction. Hence, there is at least one zero of $u^{\prime}$ between consecutive zeros of $w_{0}$.

Note that the functions $u(t), w_{0}(t)$ satisfy the following equations:

$$
\begin{gathered}
u^{\prime \prime}+\left(\mu+\frac{f(u)}{u}\right) a(t) u=0, \\
w_{0}^{\prime \prime}+\left(\mu+f^{\prime}(u)\right) a(t) w_{0}=0,
\end{gathered}
$$

respectively. Since $a(t)>0$ and $f^{\prime}(u)>f(u) / u$, by the Sturm comparison lemma, there exists at least one zero of $w_{0}$ between any two consecutive zeros of $u$. This implies that $w_{0}$ has at least $k$ zeros on $(0,1)$.

Secondly, we show (12). On the contrary, assuming $w_{0}(1)=0$, then $w_{0}$ has at least $k+2$ zeros on $[0,1]$ since $w_{0}(0)=0$. We conclude that $u^{\prime}$ has at least $k+1$ zeros on $(0,1)$. This contradicts the fact that $u^{\prime}$ has exact $k$ zeros on $(0,1)$.

Finally, we give a proper function $g$ satisfying (17). Integrating the differential equation in (17), we can choose

$$
g(t)=a^{-1 / 2}(t)
$$

In view of (H4), we conclude $g^{\prime \prime}<0$. So, the auxiliary function $g$ exists. This completes the proof.

Our main results are the following.
Theorem 5. Let (H1)-(H4) hold. Then, for fixed $k \in \mathbb{N}$ and $\mu \geq 0$, all solutions of (4) belonging to $S_{k}^{+}$(resp. $S_{k}^{-}$) lie on a unique continuous curve on which there is not any turning point. This curve starts from $\left(\lambda_{k}, 0\right)$, tends to left, and passes through the hyperplane $\{0\} \times E$. Precisely, (4) has exactly one solution belonging to $S_{k}^{+}$(resp. $\left.S_{k}^{-}\right)$for $\mu \in\left[0, \lambda_{k}\right)$ and has no solution belonging to $S_{k}^{+}\left(\right.$resp. $\left.S_{k}^{-}\right)$for $\mu \in\left[\lambda_{k}, \infty\right)$, where $\lambda_{k}$ is the $k$ th eigenvalue of (6). See Figure $1(a)$.

From Theorem 5, we immediately obtain the following theorem.

Theorem 6. Let (H1)-(H4) hold. Then (1) has a unique solution in $S_{k}^{v}$ for every $k \in \mathbb{N}$ and $v \in\{+,-\}$.

We give some lemmas for proving Theorem 5.

Lemma 7. Let $I \subset \mathbb{R}$ be a closed and bounded interval. Suppose $\left\{\left(\mu_{n}, u_{n}\right)\right\} \subset I \times S_{k}^{v}$ for some $k \geq 1$ and $v \in\{+,-\}$ is a sequence of solutions of (4). Then

$$
\mu_{n} \longrightarrow \lambda_{k}, \quad \text { if }\left\|u_{n}\right\|_{E} \longrightarrow 0 .
$$

Proof. Define $L: X \rightarrow Y$ by setting

$$
L u:=-u^{\prime \prime}, \quad u \in X .
$$

Then $L^{-1}: Y \rightarrow E$ is completely continuous. Noting that $f_{0}=0$, consider

$$
L u-\mu a(t) u=a(t) f(u)
$$

as a bifurcation problem from $u \equiv 0$. Remark 1 , Crandall and Rabinowitz theorem on bifurcation from simple eigenvalues (see [25]) and the method in [5] ensure that the result is correct.

Lemma 8. Suppose $(\mu, u) \in \mathbb{R} \times S_{k}^{v}$ for some $k \geq 1$ and $\nu \in$ $\{+,-\}$ is a solution of (4). Then $\mu<\lambda_{k}$.

Proof. Note that $u$ and $\varphi_{k}$ satisfy equations

$$
\begin{gathered}
u^{\prime \prime}+\left(\mu+\frac{f(u)}{u}\right) a(t) u=0, \\
\varphi_{k}^{\prime \prime}+\lambda_{k} a(t) \varphi_{k}=0,
\end{gathered}
$$

respectively. On the contrary, supposing $\mu \geq \lambda_{k}$, then $\mu+$ $f(u) / u \geq \lambda_{k}$ since $f(u) / u>0$ if $u \neq 0$ and $f_{0}=0$. Then by the Sturm comparison lemma, between any two consecutive zeros of $\varphi_{k}$, there exists at least one zero of $u$. This implies that $u$ has at least $k$ zeros on $(0,1)$. It is impossible.

Lemma 9. Let $J$ be a closed subinterval of $\left[0, \lambda_{k}\right)$. Then there is $b_{J}>0$ such that $\|u\|_{E} \leq b_{J}$ for every solution $(\mu, u) \in J \times S_{k}^{v}$ of (4).

Proof. Note that when $\mu \in\left[0, \lambda_{k}\right)$, we have

$$
\begin{array}{ll}
u^{\prime \prime}=-\mu a(t) u-a(t) f(u) \leq 0, & \text { if } u \geq 0, \\
u^{\prime \prime}=-\mu a(t) u-a(t) f(u) \geq 0, & \text { if } u \leq 0 .
\end{array}
$$




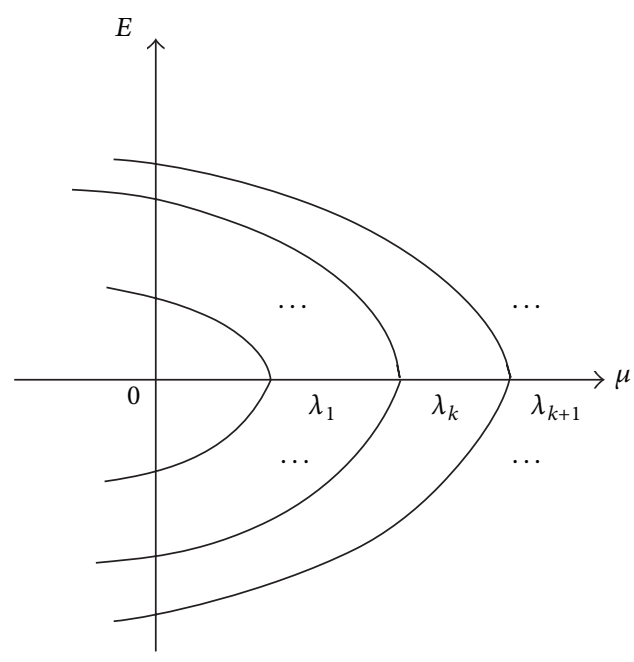

(a)

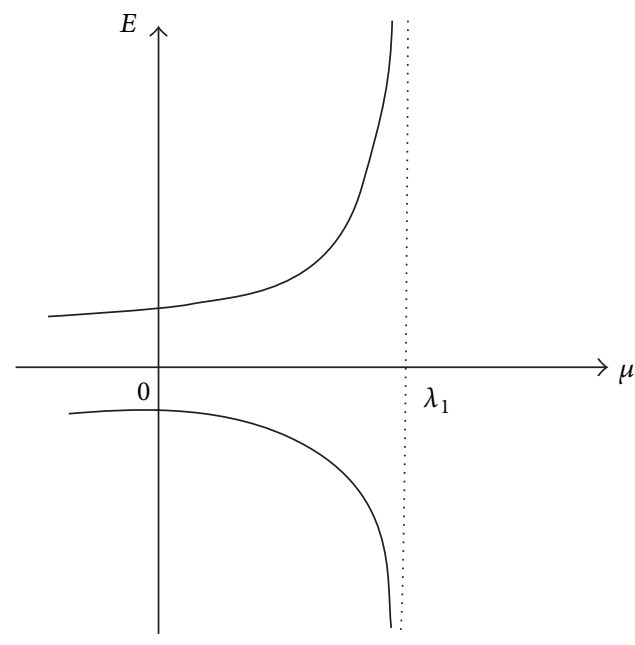

(b)

FIGURE 1: The bifurcation diagrams of solutions of (4).

Suppose on the contrary that there exists a sequence $\left\{\left(\mu_{n}, u_{n}\right)\right\}$ of solutions of (4) with $\mu_{n} \in J, u_{n} \in S_{k}^{v}$ and $\left\|u_{n}\right\|_{E} \rightarrow \infty$ as $n \rightarrow \infty$. Let

$$
0=\tau_{n}^{0}<\tau_{n}^{1}<\cdots<\tau_{n}^{k}=1
$$

denote the zeros of $u_{n}$ in $[0,1]$. Then we can choose at least one subinterval $\left(\tau_{n}^{j}, \tau_{n}^{j+1}\right) \triangleq I_{n}^{j}$ which is of length at least $1 / k$, for some $j \in\{0,1,2, \ldots, k-1\}$. Without loss of generality, we suppose $u^{\prime \prime} \leq 0$ on $I_{n}^{j}$. Moreover, we claim that $\left\{\max _{I_{n}^{j}}\left|u_{n}\right|\right\}_{n=1}^{\infty}$ is an unbounded sequence.

Assume that $\left\{\max _{I_{n}^{I}}\left|u_{n}\right|\right\}_{n=1}^{\infty}$ is uniformly bounded. Since $u_{n}$ is concave on $I_{n}^{j}, u_{n}^{\prime}$ has only one zero $\xi_{n}^{j}$ in $I_{n}^{j}$. Integrating the equation in (4), for any $t \in I_{n}^{j}$,

$$
u_{n}^{\prime}(t)=-\int_{\xi_{n}^{j}}^{t}\left(\mu_{n} u_{n}+f\left(u_{n}\right)\right) a(s) d s .
$$

It implies that $\left\{\max _{I_{n}^{j}}\left|u_{n}^{\prime}\right|\right\}_{n=1}^{\infty}$ is uniformly bounded. Then we obtain $u_{n}^{\prime}\left(\tau_{n}^{j}\right), u_{n}^{\prime}\left(\tau_{n}^{j+1}\right)$ are uniformly bounded. Consider consecutive intervals $I_{n}^{j-1}$ and $I_{n}^{j+1}$. By convexity of $u_{n}$ on $I_{n}^{j-1}$ and $I_{n}^{j+1}$ and the uniform boundedness of $u_{n}^{\prime}\left(\tau_{n}^{j}\right), u_{n}^{\prime}\left(\tau_{n}^{j+1}\right), u_{n}^{\prime}$ is uniformly bounded on $\left[\xi_{n}^{j-1}, \tau_{n}^{j}\right]$ and $\left[\tau_{n}^{j+1}, \xi_{n}^{j+1}\right]$. So,

$$
\begin{aligned}
& u_{n}\left(\xi_{n}^{j-1}\right)=\int_{\tau_{n}^{j}}^{\xi_{n}^{j-1}} u_{n}^{\prime}(s) d s, \\
& u_{n}\left(\xi_{n}^{j+1}\right)=\int_{\tau_{n}^{j+1}}^{\xi_{n}^{j+1}} u_{n}^{\prime}(s) d s
\end{aligned}
$$

are uniformly bounded. Hence, $\left\{\max \left|u_{n}\right|\right\}_{n=1}^{\infty}$ is uniformly bounded on $I_{n}^{j-1}$ and $I_{n}^{j+1}$. By some finite steps, this procedure shows that $\left\{\max \left|u_{n}\right|\right\}_{n=1}^{\infty}$ is uniformly bounded on $[0,1]$. This is a contradiction.

Taking subsequences if necessary, put

$$
\lim _{n \rightarrow \infty} \tau_{n}^{j}=\tau_{\infty}^{j}, \quad \lim _{n \rightarrow \infty} \tau_{n}^{j+1}=\tau_{\infty}^{j+1}, \quad \lim _{n \rightarrow \infty} \xi_{n}^{j}=\xi_{\infty}^{j} .
$$

Noting that $\tau_{n}^{j+1}-\tau_{n}^{j} \geq 1 / k$, then $\tau_{\infty}^{j+1}-\tau_{\infty}^{j} \geq 1 / k$. We may assume $\tau_{\infty}^{j}<\xi_{\infty}^{j}<\tau_{\infty}^{j+1}$. The case $\xi_{\infty}^{j}=\tau_{\infty}^{j}$ or $\xi_{\infty}^{j}=\tau_{\infty}^{j+1}$ can be considered similarly. In the rest of the proof, $\left\|u_{n}\right\|_{\infty}$ denotes $\max _{I_{n}^{j}}\left|u_{n}\right|$. Since $u_{n}$ is concave on $I_{n}^{j}$, for any $0<\epsilon<$ $\left(\xi_{\infty}^{j}-\tau_{\infty}^{j}\right) / 4$ small enough, there exists a constant $\sigma>0$ such that

$$
u_{n}(t) \geq \sigma\left\|u_{n}\right\|_{\infty}, \quad \forall t \in\left[\tau_{n}^{j}+\epsilon, \tau_{n}^{j+1}-\epsilon\right] .
$$

By the condition $f_{\infty}=\infty$ in (H3), there exists a real number $R_{1}>0$ such that

$$
f(u) \geq \eta u, \quad \text { for } u \geq R_{1},
$$

where $\eta>0$ can be given by

$$
\left(\mu_{n}+\eta\right) \sigma \int_{\tau_{\infty}^{j}+\epsilon}^{\tau_{\infty}^{j}+2 \epsilon} d s \int_{s}^{\tau_{\infty}^{j}+2 \epsilon} a(\tau) d \tau>1 .
$$

Since $\left\|u_{n}\right\|_{\infty} \rightarrow \infty,\left\|u_{n}\right\|_{\infty}>R_{1} / \sigma$ for sufficiently large $n$. Thus

$$
u_{n}(t) \geq \sigma\left\|u_{n}\right\|_{\infty}>R_{1}, \quad \forall t \in\left[\tau_{n}^{j}+\epsilon, \tau_{n}^{j+1}-\epsilon\right] .
$$

Moreover,

$$
\begin{aligned}
u_{n}\left(\xi_{n}^{j}\right) & =\int_{\tau_{n}^{j}}^{\xi_{n}^{j}}\left(\int_{s}^{\xi_{n}^{j}}\left[\mu_{n} a(\tau) u_{n}(\tau)+a(\tau) f\left(u_{n}(\tau)\right)\right] d \tau\right) d s \\
& \geq\left(\mu_{n}+\eta\right) \int_{\tau_{\infty}^{j}+\epsilon}^{\tau_{\infty}^{j}+2 \epsilon}\left(\int_{s}^{\tau_{\infty}^{j}+2 \epsilon}\left[a(\tau) u_{n}(\tau)\right] d \tau\right) d s \\
& \geq\left\|u_{n}\right\|_{\infty}\left(\mu_{n}+\eta\right) \sigma \int_{\tau_{\infty}^{j}+\epsilon}^{\tau_{\infty}^{j}+2 \epsilon} d s \int_{s}^{\tau_{\infty}^{j}+2 \epsilon} a(\tau) d \tau
\end{aligned}
$$

Hence,

$$
\left\|u_{n}\right\|_{\infty} \geq\left\|u_{n}\right\|_{\infty}\left(\mu_{n}+\eta\right) \sigma \int_{\tau_{\infty}^{j}+\epsilon}^{\tau_{\infty}^{j}+2 \epsilon} d s \int_{s}^{\tau_{\infty}^{j}+2 \epsilon} a(\tau) d \tau .
$$


It follows that

$$
\left(\mu_{n}+\eta\right) \sigma \int_{\tau_{\infty}^{j}+\epsilon}^{\tau_{\infty}^{j}+2 \epsilon} d s \int_{s}^{\tau_{\infty}^{j}+2 \epsilon} a(\tau) d \tau \leq 1 .
$$

This contradicts (32). This ends the proof.

Suppose there exits a nontrivial solution $\left(\mu_{0}, u_{0}\right)$ of (4) with $\mu_{0} \geq 0$. It follows from Lemma 3 that $u_{0} \in S_{k}^{v}$ for some $k \geq 1$ and $v \in\{+,-\}$. Meanwhile, from the implicit function theorem and Lemma 4, we have that all solutions of (4) in $\mathbb{R} \times E$ near $\left(\mu_{0}, u_{0}\right)$ lie on a $C^{1}$ curve passing through $\left(\mu_{0}, u_{0}\right)$ and parameterized by $\mu$. We denote the local curve by $\Gamma_{0}$. Then, we have the following lemma.

Lemma 10. $\Gamma_{0} \subset \mathbb{R} \times S_{k}^{\nu}$.

Proof. This can be obtained by Lemma 3 and the fact that $S_{k}^{v}$ is open.

Lemma 11. $\Gamma_{0}$ can be continued on the interval $\left[0, \lambda_{k}\right)$. Moreover, there is a constant $c>0$ such that

$$
\lim _{\mu \rightarrow 0}\|u\|_{E}=c>0, \quad \lim _{\mu \rightarrow \lambda_{k}}\|u\|_{E}=0 .
$$

Proof. Suppose that there exist a sequence $\left\{\left(\mu_{n}, u_{n}\right)\right\} \subset \Gamma_{0}$ and a small constant $0<\delta<1 / \delta$ such that $\mu_{n} \rightarrow e \in$ $\left(0, \lambda_{k}\right), n \rightarrow \infty$, and $\delta \leq\left\|u_{n}\right\|_{X} \leq 1 / \delta$ for large enough $n$. Then, after choosing a subsequence if necessary, there exists $u_{\infty} \in E$ such that $u_{n} \rightarrow u_{\infty}$ in $E$. It then follows from the equation in (4) that $u_{\infty} \in X$ and $\left(e, u_{\infty}\right)$ is a nontrivial solution of (4). Hence, by Lemma 4 and the implicit function theorem, the curve $\Gamma_{0}$ can still be continued. Meanwhile, we know $\lim _{\mu_{n} \rightarrow e}\left\|u_{n}\right\|_{E} \neq \infty$ from Lemma 9. Combining this with Lemma 8 , we have $\Gamma_{0}$ can be continued on the interval $\left[0, \lambda_{k}\right)$. Moreover, by Lemma 7 , there is a constant $c>0$ such that

$$
\lim _{\mu \rightarrow 0}\|u\|_{E}=c>0, \quad \lim _{\mu \rightarrow \lambda_{k}}\|u\|_{E}=0 .
$$

Assume $\left(0, u_{0}\right)$ is the intersection of $\Gamma_{0}$ and hyperplane $\{0\} \times E$. From Lemma $4,\left(0, u_{0}\right)$ is also a nondegenerate solution of (4) and $\left\|u_{0}\right\|_{E}>0$. Hence, $\Gamma_{0}$ can still be continued to the direction of $\mu<0$.

Proof of Theorem 5. Consider bifurcation problems (23), by the standard Crandall and Rabinowitz theorem on local bifurcation from simple eigenvalues (see [25]), for each $k \geq 1$ exactly two local curves of nontrivial solutions bifurcate from the point $\left(\lambda_{k}, 0\right)$ in $\mathbb{R} \times E$, one of which lies in $\mathbb{R} \times S_{k}^{+}$and the other in $\mathbb{R} \times S_{k}^{-}$. By the above discussion, each of these local curves can be continued at least on the interval $\left[0, \lambda_{k}\right)$. We will denote these particular curves by $\mathscr{C}_{k}^{v}$. Then $\mathscr{C}_{k}^{v} \subset \mathbb{R} \times S_{k}^{v}$ by Lemma 10. From Lemmas 4 and $11, \mathscr{C}_{k}^{v}$ can pass through the hyperplane $\{0\} \times E$ and go to the direction of $\mu<0$.

Finally, we exclude the possibility that there exists another solution curve of (4) belonging to the set $\left[0, \lambda_{k}\right) \times S_{k}^{v}$. Suppose that there exists a solution $(\mu, u) \in\left[0, \lambda_{k}\right) \times S_{k}^{v}$ but $(\mu, u) \notin \mathscr{C}_{k}^{v}$. Similarly, by continuation, we can obtain another solution curve $\Gamma \subset\left[0, \lambda_{k}\right) \times S_{k}^{v}$ such that $\lim _{\mu \rightarrow \lambda_{k}}\|u\|_{E}=0$ for $(\mu, u) \in \Gamma$. Since $\mathscr{C}_{k}^{v}$ is the unique solution curves near $\left(\lambda_{k}, 0\right)$ in $\mathbb{R} \times E$ by the standard Crandall and Rabinowitz theorem, $\Gamma$ must coincide with $\mathscr{C}_{k}^{v}$. This completes the proof.

Proof of Theorem 6. From Theorem 5 and Lemma 11, we obtain the result.

\section{The Main Results under $f_{0}=\infty, f_{\infty}=0$}

In this section, we study the exact multiplicity of constant sign, that is, positive (resp. negative) solution of (1) under the conditions

$\left(\mathrm{H} 1^{\prime}\right) f \in C(\mathbb{R}, \mathbb{R}), f(0)=0$. When $u \neq 0, f^{\prime}(u)$ exists and satisfies $f^{\prime}(u)>0 . f^{\prime}(u)$ is nondecreasing on $(-\infty, 0)$ and nonincreasing on $(0, \infty)$. For any fixed $\alpha>0$, $\int_{0}^{1} t f^{\prime}( \pm \alpha t(1-t)) d t<\infty$ and $\int_{0}^{1}(1-t) f^{\prime}( \pm \alpha t(1-$ t)) $d t<\infty$.

$\left(\mathrm{H} 2^{\prime}\right) f^{\prime}(u)<f(u) / u$, if $u \neq 0$;

$\left(\mathrm{H} 3^{\prime}\right) f_{0}=\lim _{|s| \rightarrow 0}(f(s) / s)=\infty, f_{\infty}=$ $\lim _{|s| \rightarrow \infty}(f(s) / s)=0 ;$

$\left(\mathrm{H}^{\prime}\right) a(t) \in C^{1}[0,1]$ satisfies $a(t)>0, t \in[0,1]$.

Remark 12 . There are many functions satisfying conditions $\left(\mathrm{H1}^{\prime}\right)-\left(\mathrm{H} 3^{\prime}\right)$. For example,

$$
f(u)= \begin{cases}\sqrt{u}, & u \geq 0, \\ -\sqrt{-u}, & u<0 .\end{cases}
$$

As before, we discuss the structure of the set of solutions of the auxiliary problem (4).

Lemma 13. If $(\mu, u)$ is a solution of (4), then the zero of $u$ is simple and $u^{\prime}(0) \neq 0, u^{\prime}(1) \neq 0$. Moreover, $u \in S_{k}^{v}$ for some $k \geq$ 1 and $\nu \in\{+,-\}$.

Proof. From proportion 2.1 in [20], we can obtain, for every $t_{0} \in[0,1]$ and $\beta \in \mathbb{R}$, the initial value problem

$$
\begin{gathered}
u^{\prime \prime}+\mu a(t) u+a(t) f(u)=0, \quad t \in(0,1), \\
u\left(t_{0}\right)=0, \quad u^{\prime}\left(t_{0}\right)=\beta
\end{gathered}
$$

has a unique solution. Particularly, it is true for the case $f_{0}=\infty$. Thus, $u \in S_{k}^{v}$ for some $k \geq 1$ and $v \in\{+,-\}$ and $u^{\prime}(0) \neq 0, u^{\prime}(1) \neq 0$.

Lemma 14. For $v \in\{+,-\}$, if $(\mu, u) \in S_{1}^{v}$ is a solution of (4), then $\mu<\lambda_{1}$, where $\lambda_{1}$ is the first eigenvalue of (6).

Proof. Note that $u$ and $\varphi_{1}$ satisfy, respectively, the equations

$$
\begin{gathered}
u^{\prime \prime}+\left(\mu+\frac{f(u)}{u}\right) a(t) u=0 \\
\varphi_{1}^{\prime \prime}+\lambda_{1} a(t) \varphi_{1}=0
\end{gathered}
$$

On the contrary, assume $\mu \geq \lambda_{1}$. Noting that for $u \neq 0$ we have $f(u) / u>0$ and $f_{0}=\infty$, then $\mu+f(u) / u \geq \lambda_{1}$. By the Sturm 
comparison lemma, there exists at least one zero of $u$ between any two consecutive zeros of $\varphi_{1}$. This implies that $u$ has at least one zeros on $(0,1)$. It is impossible.

Lemma 15. Let $I \subset \mathbb{R}$ be a bounded and closed interval and $\lambda_{1} \in I$. For $v \in\{+,-\}$, if $\left(\mu_{n}, u_{n}\right) \in S_{1}^{\nu}(n=1,2, \ldots)$ are solutions of (4) and $\mu_{n} \in I$, then

$$
\mu_{n} \longrightarrow \lambda_{1} \text { as }\left\|u_{n}\right\|_{E} \longrightarrow \infty \text {. }
$$

Proof. Note that $f_{\infty}=0$ consider the bifurcation problems (23) from infinity. By using Remark 1 and standard Rabinowitz bifurcation theorem from simple eigenvalue in [26] and the proof method in [5], we can obtain the result.

From Lemma 15, we have the following lemma.

Lemma 16. Let $\bar{J} \subset\left[0, \lambda_{1}\right)$ be a closed interval. Then there exists $b_{\bar{J}}>0$ such that

$$
\|u\|_{E} \leq b_{\bar{J}}
$$

for every solution $(\mu, u) \in \bar{J} \times S_{1}^{v}$ of $(4)$.

Lemma 17. Let $\tilde{J} \subset\left[0, \lambda_{1}\right)$ be a closed interval. Then there exists $c_{\tilde{J}}>0$ such that

$$
\|u\|_{E} \geq \mathcal{c}_{\tilde{J}}
$$

for each solution $(\mu, u) \in \tilde{J} \times S_{1}^{v}$ of $(4)$.

Proof. Without loss of generality, we select $S_{1}^{+}$to be discussed. When $\mu \in\left[0, \lambda_{1}\right)$, since $(\mu, u)$ is a positive solution of $(4)$, we have

$$
u^{\prime \prime}=-\mu a(t) u-a(t) f(u) \leq 0 .
$$

On the contrary, suppose there exists a sequence of solutions of (4) $\left\{\left(\mu_{n}, u_{n}\right)\right\} \subset \tilde{J} \times S_{1}^{+}$satisfying

$$
\mu_{n} \in J, \quad\left\|u_{n}\right\|_{E} \longrightarrow 0, \quad \text { as } n \longrightarrow \infty \text {. }
$$

Noting that $u_{n}$ is concave on the interval $(0,1)$, then $u_{n}^{\prime}$ has only one zero in $(0,1)$, denoted by $\xi_{n}$. Integrating

$$
u_{n}^{\prime \prime}+\mu_{n} a(t) u_{n}+a(t) f\left(u_{n}\right)=0, \quad t \in(0,1),
$$

we get

$$
u_{n}^{\prime}(t)=-\int_{\xi_{n}}^{t}\left(\mu_{n} u_{n}+f\left(u_{n}\right)\right) a(s) d s .
$$

Taking subsequences if necessary, let

$$
\lim _{n \rightarrow \infty} \xi_{n}=\xi_{\infty}
$$

Without loss of generality, suppose $0<\xi_{\infty}<1$. For the case $\xi_{\infty}=0$ or $\xi_{\infty}=1$, the proof is similar. Since $u_{n}$ is concave on the interval $(0,1)$, for $0<\epsilon<\xi_{\infty} / 4$ small enough, there exists a constant $\bar{\sigma}>0$ such that for large $n$

$$
u_{n}(t) \geq \bar{\sigma}\left\|u_{n}\right\|_{\infty}, \quad \forall t \in[\epsilon, 1-\epsilon] .
$$

Since $f_{0}=\infty$, there exists constant $R_{1}>0$ such that

$$
f(u) \geq \bar{\eta} u, \quad \text { as } \quad|u| \leq R_{1},
$$

where $\bar{\eta}>0$ satisfies

$$
\left(\mu_{n}+\bar{\eta}\right) \bar{\sigma} \int_{\epsilon}^{2 \epsilon} d s \int_{s}^{2 \epsilon} a(\tau) d \tau>1
$$

Since $\left\|u_{n}\right\|_{E} \rightarrow 0$, we have $\left\|u_{n}\right\|_{\infty} \rightarrow 0$. Thus, for large enough $n,\left\|u_{n}\right\|_{\infty}<R_{1}$. And for every $t \in[\epsilon, 1-\epsilon]$, we have

$$
\bar{\sigma}\left\|u_{n}\right\|_{\infty} \leq u_{n}(t) \leq\left\|u_{n}\right\|_{\infty} .
$$

Moreover,

$$
\begin{aligned}
u_{n}\left(\xi_{n}\right) & =\int_{0}^{\xi_{n}}\left(\int_{s}^{\xi_{n}}\left[\mu_{n} a(\tau) u_{n}(\tau)+a(\tau) f\left(u_{n}(\tau)\right)\right] d \tau\right) d s \\
& \geq\left(\mu_{n}+\bar{\eta}\right) \int_{\epsilon}^{2 \epsilon}\left(\int_{s}^{2 \epsilon}\left[a(\tau) u_{n}(\tau)\right] d \tau\right) d s \\
& \geq\left\|u_{n}\right\|_{\infty}\left(\mu_{n}+\bar{\eta}\right) \bar{\sigma} \int_{\epsilon}^{2 \epsilon} d s \int_{s}^{2 \epsilon} a(\tau) d \tau .
\end{aligned}
$$

Therefore,

$$
\left\|u_{n}\right\|_{\infty} \geq\left\|u_{n}\right\|_{\infty}\left(\mu_{n}+\bar{\eta}\right) \bar{\sigma} \int_{\epsilon}^{2 \epsilon} d s \int_{s}^{2 \epsilon} a(\tau) d \tau .
$$

From this, we have

$$
\left(\mu_{n}+\eta\right) \sigma \int_{\epsilon}^{2 \epsilon} d s \int_{s}^{2 \epsilon} a(\tau) d \tau \leq 1 .
$$

It contradicts (52). This ends the proof.

Lemma 18. Let $\mu \in\left[0, \lambda_{1}\right)$ and $(\mu, u) \in \mathbb{R} \times S_{1}^{v}$ be a solution of $(4)$. Then $(\mu, u)$ is nondegenerate.

Proof. It is sufficient to prove that (5) has only trivial solution. Consider the initial value problem

$$
\begin{gathered}
w^{\prime \prime}+\mu a(t) w+a(t) f_{u}(u(t)) w=0, \quad t \in(0,1), \\
w(0)=0, \quad w^{\prime}(0)=\beta \neq 0,
\end{gathered}
$$

where $f_{u}$ denotes the derivative of $f$ to $u . \beta \in \mathbb{R}$ is a constant. First, we prove (57) has a unique solution. From Lemma 3.1 and its proof, Lemma 3.2 in [3], we need to show

$$
\int_{0}^{1} t\left|\mu+f_{u}(u(t))\right| a(t) d t<\infty,
$$

$$
\int_{0}^{1}(1-t)\left|\mu+f_{u}(u(t))\right| a(t) d t<\infty .
$$

It is sufficient to prove

$$
\begin{gathered}
\int_{0}^{1} t f_{u}(u(t)) d t<\infty, \\
\int_{0}^{1}(1-t) f_{u}(u(t)) d t<\infty .
\end{gathered}
$$


Noting that for every $t \in(0,1)$, if $u(t)>0$, then $u^{\prime \prime}(t)<0$; if $u(t)<0$, then $u^{\prime \prime}(t)>0$. Suppose $(\mu, u) \in \mathbb{R} \times S_{1}^{+}$(when $(\mu, u) \in \mathbb{R} \times S_{1}^{-}$, the proof is similar). Then there must exist $\alpha>0$ such that $u(t)>\alpha t(1-t)>0$, for all $t \in(0,1)$. By condition $\left(\mathrm{Hl}^{\prime}\right)$, we have

$$
f_{u}(u(t))<f_{u}(\alpha t(1-t)), \quad \forall t \in(0,1) .
$$

It follows that

$$
\begin{gathered}
\int_{0}^{1} t f_{u}(u(t)) d t<\int_{0}^{1} t f_{u}(\alpha t(1-t)) d t<\infty, \\
\int_{0}^{1}(1-t) f_{u}(u(t)) d t<\int_{0}^{1}(1-t) f_{u}(\alpha t(1-t)) d t<\infty .
\end{gathered}
$$

From Lemma 3.2 in [3], (57) has a unique solution. When $\beta=1$, we denote the solution of (57) by $w_{0}$. Then, for every solution $w$ of (57), there exists a constant $d \in \mathbb{R}$ such that $w=d w_{0}$.

We claim that

$$
w_{0}(1) \neq 0 \text {. }
$$

If (62) holds, then we immediately have $w(1)=0$ if and only if $d=0$. That is to say, $w \equiv 0$. Then we will finish the proof.

Note that $u(t), w_{0}(t)$ satisfy the following equations:

$$
\begin{gathered}
u^{\prime \prime}+\left(\mu+\frac{f(u)}{u}\right) a(t) u=0, \\
w_{0}^{\prime \prime}+\left(\mu+f^{\prime}(u)\right) a(t) w_{0}=0,
\end{gathered}
$$

respectively. Since $a(t)>0, f^{\prime}(u)<f(u) / u, u \neq 0$, by the Sturm comparison lemma, there exists at least one zero of $u$ between any two consecutive zeros of $w_{0}$. If (62) does not hold, then

$$
w_{0}(1)=0 \text {. }
$$

Complying this with $w_{0}(0)=0$, we obtain there exists at least one zero of $u$ in the interval $(0,1)$. This contradicts that $u$ is a positive (resp. negative) solution of (4). The proof is ended.

Suppose $\mu_{0} \geq 0$ and $\left(\mu_{0}, u_{0}\right)$ is a positive (resp. negative) solution of (4). For $v \in\{+,-\}$, we have $u_{0} \in S_{1}^{\nu}$. By Lemma 18 and the implicit function theorem, all solutions of (4) near $\left(\mu_{0}, u_{0}\right)$ lie on a unique curve which passes through $\left(\mu_{0}, u_{0}\right)$ and is parameterized by $\mu$. Denote the curve by $\tilde{\Gamma}_{0}$. Then we have the following lemma.

Lemma 19. $\tilde{\Gamma}_{0} \subset \mathbb{R} \times S_{1}^{\nu}$.

Proof. Note that $S_{1}^{\nu}$ is an open set. This together with Lemma 13 implies the conclusion.

Lemma 20. $\tilde{\Gamma}_{0}$ can be continued on the interval $\left[0, \lambda_{1}\right)$ and there exists $h>0$ such that

$$
\lim _{\mu \rightarrow 0}\|u\|_{E}=h>0, \quad \lim _{\mu \rightarrow \lambda_{1}}\|u\|_{E}=\infty .
$$

Proof. From Lemmas 14-18 and the implicit function theorem, we can prove the result by using similar method of proving Lemma 11. We omit it.

Suppose $\left(0, u_{0}\right)$ is the intersection point of $\tilde{\Gamma}_{0}$ and hyperplane $\{0\} \times E$. From Lemma $18,\left(0, u_{0}\right)$ is also a nondegenarate solution of (4) and $\|u\|_{E}=h>0$. Hence, $\tilde{\Gamma}_{0}$ can be continued to the direction of $\mu<0$.

Theorem 21. Let $\left(\mathrm{Hl}^{\prime}\right),\left(\mathrm{H} 2^{\prime}\right),\left(H 3^{\prime}\right)$, and $\left(\mathrm{H}^{\prime}\right)$ hold. Then for $\mu \geq 0$ all solutions of (4) belonging to $S_{1}^{+}$(resp. $S_{1}^{-}$) lie on a unique continuous curve on which there is not any turning point. This curve bifurcates from $\left(\lambda_{1}, \infty\right)$, tends to left, and passes through the hyperplane $\{0\} \times$ E. Precisely, (4) has exactly one positive (resp. negative) solution if $\mu \in\left[0, \lambda_{1}\right)$ and has no positive (resp. negative) solution for $\mu \in\left[\lambda_{1}, \infty\right)$, where $\lambda_{1}$ is the first eigenvalue of the linear problem (6). See Figure 1(b).

Proof. With the uniqueness of local curve of solutions bifurcating from infinity [26], the proof is similar to that of Theorem 5. For simplicity, we omit it.

From Lemma 20 and Theorem 21, we immediately obtain the following.

Theorem 22. Let $\left(H 1^{\prime}\right),\left(H 2^{\prime}\right),\left(H 3^{\prime}\right)$, and $\left(H 4^{\prime}\right)$ hold. Then (1) has a unique positive (resp. negative) solution.

\section{Acknowledgments}

This paper is supported by the NFSC (10971139), China Postdoctoral Fund (no. 2011M500615), Innovation Program of Shanghai Municipal Education Commission (no. 11YZ225).

\section{References}

[1] A. Ambrosetti and P. Hess, "Positive solutions of asymptotically linear elliptic eigenvalue problems," Journal of Mathematical Analysis and Applications, vol. 73, no. 2, pp. 411-422, 1980.

[2] L. H. Erbe and H. Wang, "On the existence of positive solutions of ordinary differential equations," Proceedings of the American Mathematical Society, vol. 120, no. 3, pp. 743-748, 1994.

[3] H. Asakawa, "Nonresonant singular two-point boundary value problems," Nonlinear Analysis. Theory, Methods \& Applications, vol. 44, no. 6, pp. 791-809, 2001.

[4] R. Ma and B. Thompson, "Multiplicity results for secondorder two-point boundary value problems with superlinear or sublinear nonlinearities," Journal of Mathematical Analysis and Applications, vol. 303, no. 2, pp. 726-735, 2005.

[5] R. Ma and B. Thompson, "Nodal solutions for nonlinear eigenvalue problems," Nonlinear Analysis. Theory, Methods \& Applications, vol. 59, no. 5, pp. 707-718, 2004.

[6] J. Chamberlain, L. Kong, and Q. Kong, "Nodal solutions of boundary value problems with boundary conditions involving Riemann-Stieltjes integrals," Nonlinear Analysis. Theory, Methods \& Applications, vol. 74, no. 6, pp. 2380-2387, 2011.

[7] T. Jankowski, "Existence of positive solutions to third order differential equations with advanced arguments and nonlocal boundary conditions," Nonlinear Analysis. Theory, Methods \& Applications, vol. 75, no. 2, pp. 913-923, 2012. 
[8] T. Ouyang and J. Shi, "Exact multiplicity of positive solutions for a class of semilinear problem-II," Journal of Differential Equations, vol. 158, no. 1, pp. 94-151, 1999.

[9] B. Xu, "Exact multiplicity and global structure of solutions for a class of semilinear elliptic equations," Journal of Mathematical Analysis and Applications, vol. 341, no. 2, pp. 783-790, 2008.

[10] Y. Wang, Y. Wang, and J. Shi, "Exact multiplicity of solutions to a diffusive logistic equation with harvesting," Applied Mathematics and Computation, vol. 216, no. 5, pp. 1531-1537, 2010.

[11] T. Adamowicz and P. Korman, "Remarks on time map for quasilinear equations," Journal of Mathematical Analysis and Applications, vol. 376, no. 2, pp. 686-695, 2011.

[12] K.-C. Hung and S.-H. Wang, "Classification and evolution of bifurcation curves for a multiparameter $p$-Laplacian Dirichlet problem," Nonlinear Analysis. Theory, Methods \& Applications, vol. 74, no. 11, pp. 3589-3598, 2011.

[13] J. García-Melián, "Multiplicity of positive solutions to boundary blow-up elliptic problems with sign-changing weights," Journal of Functional Analysis, vol. 261, no. 7, pp. 1775-1798, 2011.

[14] J. Shi, "Exact multiplicity of solutions to superlinear and sublinear problems," Nonlinear Analysis. Theory, Methods \& Applications, vol. 50, no. 5, pp. 665-687, 2002.

[15] R. Bari and B. P. Rynne, "Solution curves and exact multiplicity results for $2 \mathrm{mth}$ order boundary value problems," Journal of Mathematical Analysis and Applications, vol. 292, no. 1, pp. 1722, 2004.

[16] P. Korman, "Curves of sign-changing solutions for semilinear equations," Nonlinear Analysis. Theory, Methods \& Applications, vol. 51, no. 5, pp. 801-820, 2002.

[17] H. Pan and R. Xing, "Time maps and exact multiplicity results for one-dimensional prescribed mean curvature equations-II," Nonlinear Analysis. Theory, Methods \& Applications, vol. 74, no. 11, pp. 3751-3768, 2011.

[18] Y. An and R. Ma, "Exact multiplicity of solutions for a class of two-point boundary value problems," Electronic Journal of Differential Equations, no. 27, article 7, 2010.

[19] H. Pan and R. Xing, "Time maps and exact multiplicity results for one-dimensional prescribed mean curvature equations," Nonlinear Analysis. Theory, Methods \& Applications, vol. 74, no. 4, pp. 1234-1260, 2011.

[20] Y. Naito and S. Tanaka, "On the existence of multiple solutions of the boundary value problem for nonlinear second-order differential equations," Nonlinear Analysis. Theory, Methods \& Applications, vol. 56, no. 6, pp. 919-935, 2004.

[21] Q. Kong, "Existence and nonexistence of solutions of secondorder nonlinear boundary value problems," Nonlinear Analysis. Theory, Methods \& Applications, vol. 66, no. 11, pp. 2635-2651, 2007.

[22] L. Kong and Q. Kong, "Nodal solutions of second order nonlinear boundary value problems," Mathematical Proceedings of the Cambridge Philosophical Society, vol. 146, no. 3, pp. 747763, 2009.

[23] V. Anuradha and R. Shivaji, "Sign changing solutions for a class of superlinear multi-parameter semi-positone problems," Nonlinear Analysis. Theory, Methods \& Applications, vol. 24, no. 11, pp. 1581-1596, 1995.

[24] P. Korman and T. Ouyang, "Solution curves for two classes of boundary-value problems," Nonlinear Analysis. Theory, Methods \& Applications, vol. 27, no. 9, pp. 1031-1047, 1996.

[25] M. G. Crandall and P. H. Rabinowitz, "Bifurcation from simple eigenvalues," Journal of Functional Analysis, vol. 8, pp. 321-340, 1971.
[26] P. H. Rabinowitz and H. Paul, "On bifurcation from infinity," Journal of Differential Equations, vol. 14, pp. 462-475, 1973. 


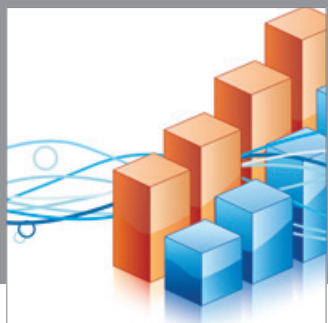

Advances in

Operations Research

mansans

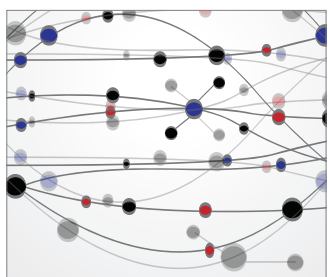

The Scientific World Journal
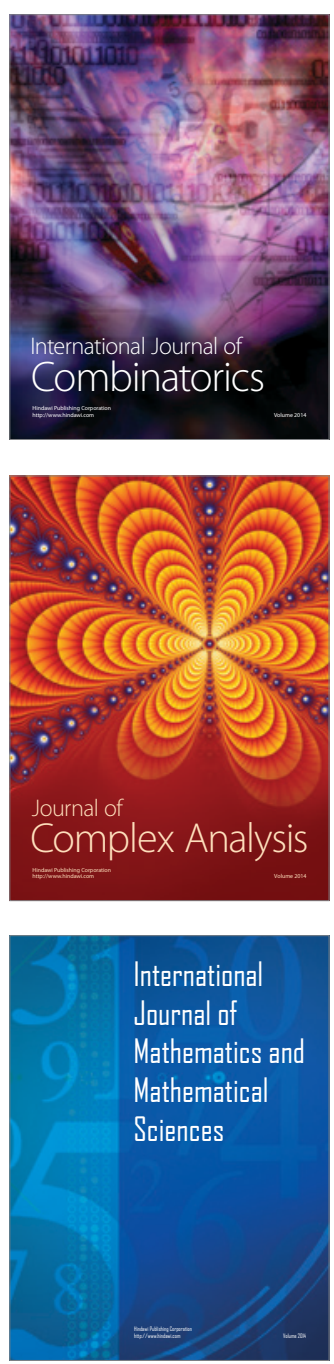
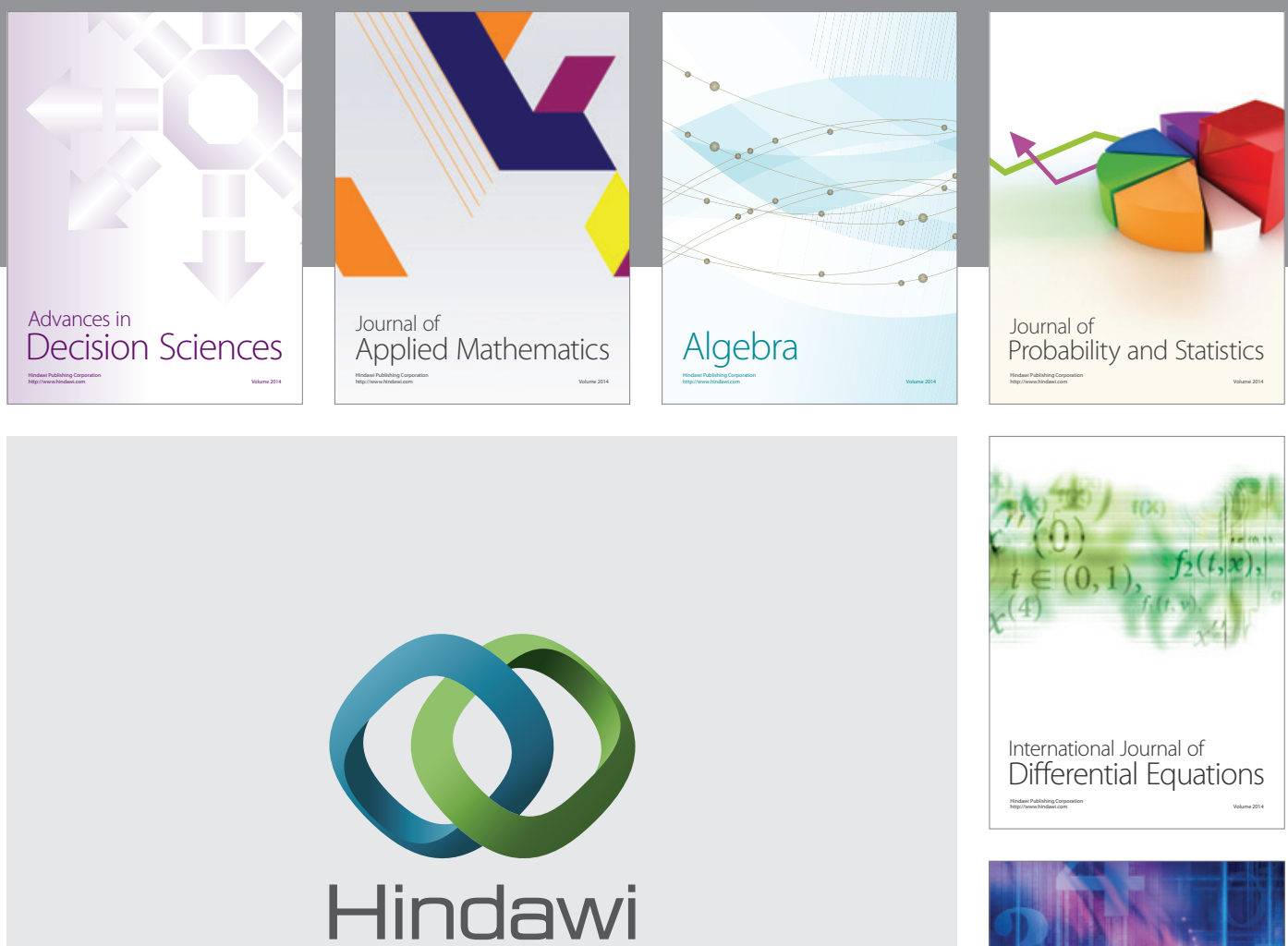

Submit your manuscripts at http://www.hindawi.com
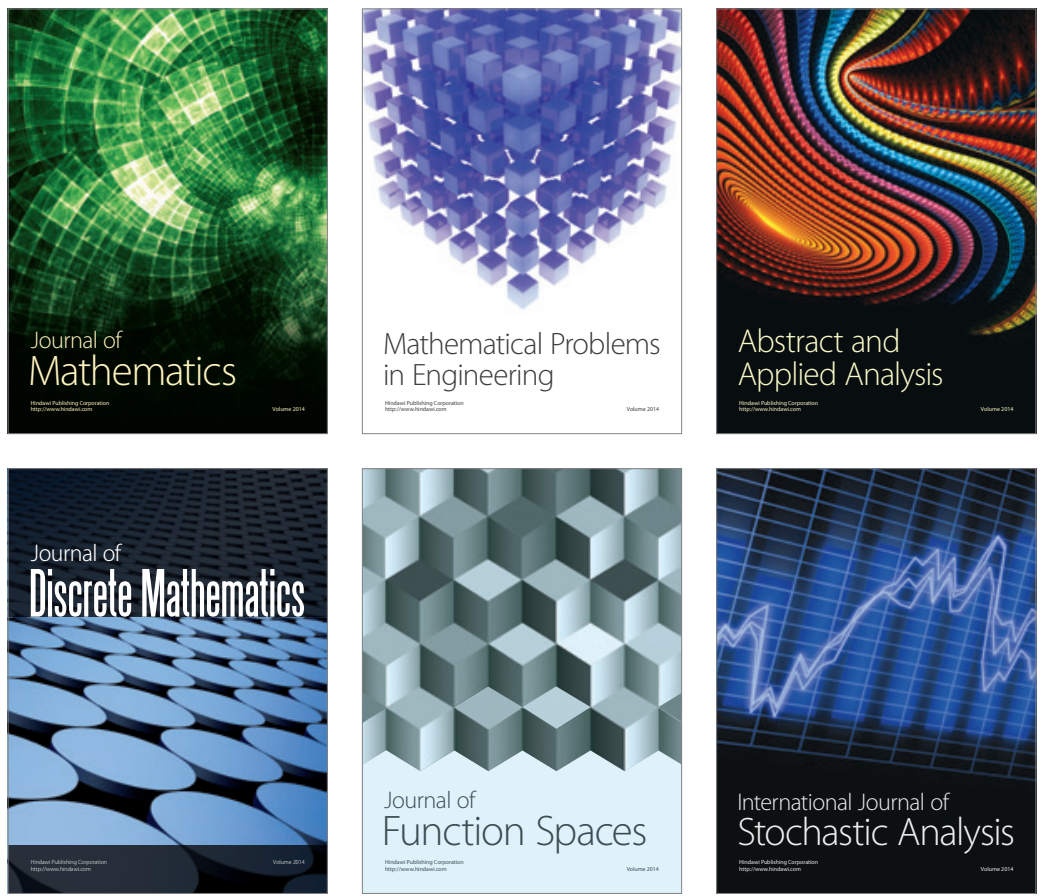

Journal of

Function Spaces

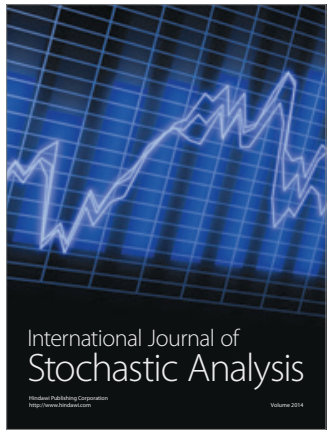

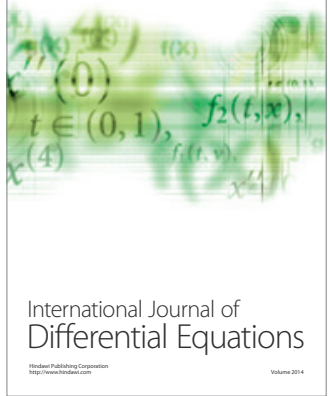
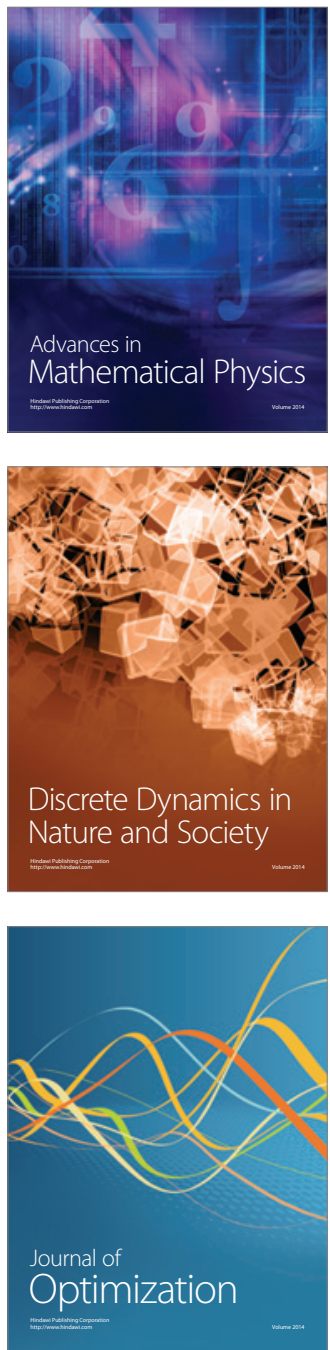